The LHCb Vertex Locator (VELO) Pixel Detector Upgrade

This content has been downloaded from IOPscience. Please scroll down to see the full text. 2017 JINST 12 C01013

(http://iopscience.iop.org/1748-0221/12/01/C01013)

View the table of contents for this issue, or go to the journal homepage for more

Download details:

IP Address: 128.141.212.91

This content was downloaded on 27/01/2017 at 10:51

Please note that terms and conditions apply.

You may also be interested in:

The LHCb VERTEX LOCATOR performance and VERTEX LOCATOR upgrade

P Rodríguez Pérez

Upgrade of the LHCb Vertex Locator

A Leflat

The LHCb Vertex Locator - performance and radiation damage

A Oblakowska-Mucha

Physics benchmarks of the VELO upgrade

L. Eklund

The upgrade of the LHCb Vertex Locator

T. Bird

Performance of the LHCb Vertex Locator

P M Bjørnstad

Calibration and Performance Monitoring of the LHCb Vertex Locator

Karol Hennessy

A PCle Gen3 based readout for the LHCb upgrade

M Bellato, G Collazuol, I D'Antone et al. 
PIXEL 2016 INTERNATIONAL WORKSHOP

September 5 - September 9, 2016

Sestri LeVante, Genova, Italy

\section{The LHCb Vertex Locator (VELO) Pixel Detector Upgrade}

\section{E. Buchanan on behalf of $\mathrm{LHCb}$ collaboration}

University of Bristol, H.H. Wills Physics Laboratory, Bristol, United Kingdom

E-mail: emma.buchanan@bristol.ac.uk

AbstRact: The LHCb experiment is designed to perform high-precision measurements of $\mathrm{CP}$ violation and the decays of beauty and charm hadrons at the Large Hadron Collider (LHC) at CERN. There is a planned upgrade during Long Shutdown 2 (LS2), expected in 2019, which will allow the detector to run at higher luminosities by transforming the entire readout to a trigger-less system. This will include a substantial upgrade of the Vertex Locator (VELO), the silicon tracker that surrounds the $\mathrm{LHCb}$ interaction region. The VELO is moving from silicon strip technology to hybrid pixel sensors, where silicon sensors are bonded to VeloPix ASICs. Sensor prototypes have undergone rigorous testing using the Timepix 3 Telescope at the SPS, CERN. The main components of the upgrade are summarised and testbeam results presented.

Keywords: Particle tracking detectors; Particle tracking detectors (Solid-state detectors); Radiation-hard detectors; Si microstrip and pad detectors 


\section{Contents}

1 Introduction 1

2 The Vertex Locator (VELO) 2

2.1 Current VELO 2

2.2 Upgraded VELO 2

2.3 Current Status 3

3 Sensor Prototype Testing 3

3.1 Sensor Prototypes and Requirements 3

3.2 Timepix3 Telescope and SPS Testbeams 4

3.3 Testbeam Analysis 4

3.3.1 Charge Collection 5

3.3.2 Charge Collection Efficiency 5

$\begin{array}{lll}\text { 3.3.3 Spatial Resolution } & 7\end{array}$

4 Conclusion $\quad 8$

\section{Introduction}

The Large Hadron Collider Beauty (LHCb) Experiment is a single arm spectrometer searching for new physics through measuring $\mathrm{CP}$ violation and rare decays of heavy flavour hadrons. The detector design is optimised for the study of particles containing $\mathrm{b}$ and $\mathrm{c}$ quarks, utilising a tracking system, two RICH detectors for particle identification, electromagnetic and hadronic calorimeters and a muon system [1]. The experiment has been running successfully since 2009, producing a variety of results. During Long Shutdown 2 (LS2), LHCb will be upgraded to further the physics performance [2]. Increasing statistics will allow precision measurements of very rare decays and greater sensitivity to a variety of new physics models. Currently LHCb runs at a luminosity of $4 \times 10^{32} \mathrm{~cm}^{-2} \mathrm{~s}^{-1}$ and on average has 1.5 interactions per bunch crossing. The luminosity is limited by the trigger, which consists of both a hardware and software level, where the former has a readout limit of $1 \mathrm{MHz}$ [3]. The luminosity is expected to increase to $2 \times 10^{33} \mathrm{~cm}^{-2} \mathrm{~s}^{-1}$. To run at this luminosity, the hardware trigger will be replaced with a fully flexible software trigger with a readout of $40 \mathrm{MHz}$ and an interaction rate ranging from 3.6-7.2 per bunch crossing [4]. To achieve this, all of the LHCb front end readout will be replaced and certain sub-detectors will be upgraded to improve tracking and withstand the increased luminosity. This is especially true for the Vertex Locator (VELO) [5], the silicon detector that surrounds the interaction region of LHCb reconstructing primary and secondary vertices. The close proximity of the VELO to the beam line means that the radiation environment will be much harsher and the increased rate will require more sophisticated electronics. This paper describes the main components of the VELO upgrade and the 
challenges faced, focusing on the sensor prototypes which have been characterised in a testbeam environment and presenting a range of the data analysis.

\section{The Vertex Locator (VELO)}

\subsection{Current VELO}

The current VELO consists of 42 semi-circular modules equipped with two $300 \mu$ m thick silicon strip sensors, measuring $r$ and $\phi$ coordinates [6]. The sensors are mounted on either side of a thermally conductive spine that provides evaporative $\mathrm{CO}_{2}$ cooling to the sensors and readout ASICs (figure 1(left)). The detector is separated into two retractable halves located inside the LHC beam pipe, separated from the primary vacuum by a $300 \mu \mathrm{m}$ thick RF foil (figure 1(right)). During stable beams, the two halves close creating a full geometrical coverage around the interaction region with the closest active strip only $8.2 \mathrm{~mm}$ from the beam line.
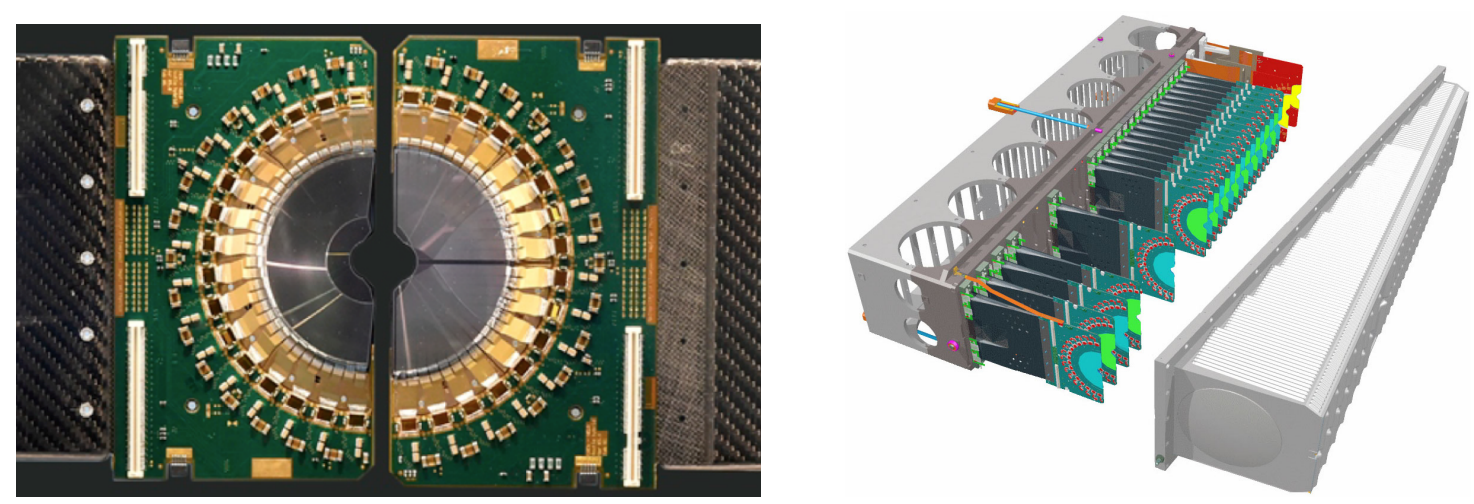

Figure 1. (left) Photograph of two prototypes of the currently used VELO strip detector modules. (right) Illustration of one half of the current VELO, depicting the semi-circular modules, support structure and RF foil.

\subsection{Upgraded VELO}

For the upgraded VELO the main mechanical infrastructure will remain. The largest change is the move from silicon strip sensors to hybrid pixel sensors [7]. A $200 \mu \mathrm{m}$ n-on-p silicon sensor will be bump bonded to a custom made VeloPix ASIC. The VeloPix, based on the Timepix3, consists of $256 \times 256$ pixels with a pixel pitch of $55 \mu \mathrm{m}$. It allows very high data rates ( $800 \mathrm{Mhits} / \mathrm{s}$ ) with a binary, data driven readout, meaning that every hit is time stamped, labelled and immediately sent off the chip. The module layout will change to a new L shape geometry with four sensors, two on each side. Each sensor consists of three Velopix ASICs connected to the same silicon sensor, referred to as a triple. An illustration of the upgraded VELO can be seen in figure 2 (left). The backbone of the module will be a $400 \mu \mathrm{m}$ thick silicon substrate that both supports the sensors (and corresponding readout) and incorporates a micro-channel cooling system. Trenches $120 \mu \mathrm{m}$ deep are etched into the silicon substrate, allowing evaporative $\mathrm{CO}_{2}$ to flow through the channels (figure 2(right)). There will be 19 channels under each ASIC, maintaining the temperature to about $\sim-30^{\circ} \mathrm{C}$. A new RF box has been designed to accommodate the new module geometry and allow 
a closer proximity to the beam line, the closest active pixel will be at $5.1 \mathrm{~mm}$ during nominal operations. The aluminium foil will have a reduced thickness of $250 \mu \mathrm{m}$ to reduce the material budget and hence suppress multiple scattering effects.
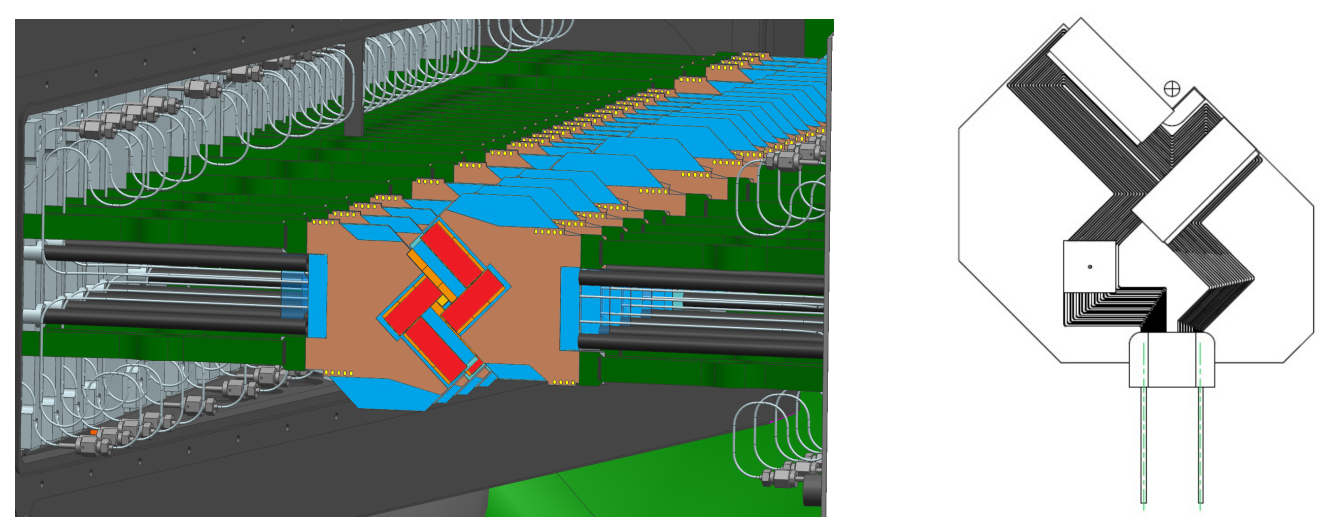

Figure 2. (left) An illustration of the Upgraded VELO closed during stable beams. Two triple sensors (red) can be seen on each module mounted on the micro-channel cooling substrate (blue). An additional two sensors are mounted on the other side of the module to provide complete coverage. (right) The silicon substrate that both supports the sensors and incorporates the micro-channel cooling.

\subsection{Current Status}

The upgrade is progressing well and the new VELO will be installed during Long Shutdown 2 (LS2) which is planned to start in 2019. Currently we have half size prototypes of both the RF foil and the micro-channel cooling which are close to fulfilling our requirements. The VeloPix ASIC design was submitted in May 2016 and arrived in September 2016 and testing has begun. The sensor prototype testing has been on going since 2014 and the final sensor design will be decided by the end of 2016 .

\section{Sensor Prototype Testing}

\subsection{Sensor Prototypes and Requirements}

A range of prototype sensors has been tested from manufacturers Hamamatsu (HPK) and Micron. HPK provided n-on-p sensors with $200 \mu \mathrm{m}$ thickness, an implant width of $35 \mu \mathrm{m}$ or $39 \mu \mathrm{m}$ and a inactive edge of $450 \mu \mathrm{m}$ or $600 \mu \mathrm{m}$. Micron provided both n-on-p and n-on-n sensors, with the former having a thickness of $200 \mu \mathrm{m}$ and the latter $150 \mu \mathrm{m}$. Both sensors have an implant width of $36 \mu \mathrm{m}$ and guard ring designs varied from $150 \mu \mathrm{m}$ to $450 \mu \mathrm{m}$. For testing purposes the sensors were bump bonded to the Timepix3 ASIC (figure 3), a predecessor to the VeloPix. The Timepix3 is an excellent general purpose ASIC providing a fast ToT readout [8].

For good physics performance there are a few benchmarks that the sensors must fulfill. Most importantly the sensors must be radiation tolerant as radiation damage degrades the sensor performance. The innermost tip of the sensors are required to withstand a maximum fluence of $8 \times 10^{15}$ $1 \mathrm{MeVn}_{\mathrm{eq}} \mathrm{cm}^{-2}$, the expected lifetime fluence for the VELO. Radiation induced trapping centres 


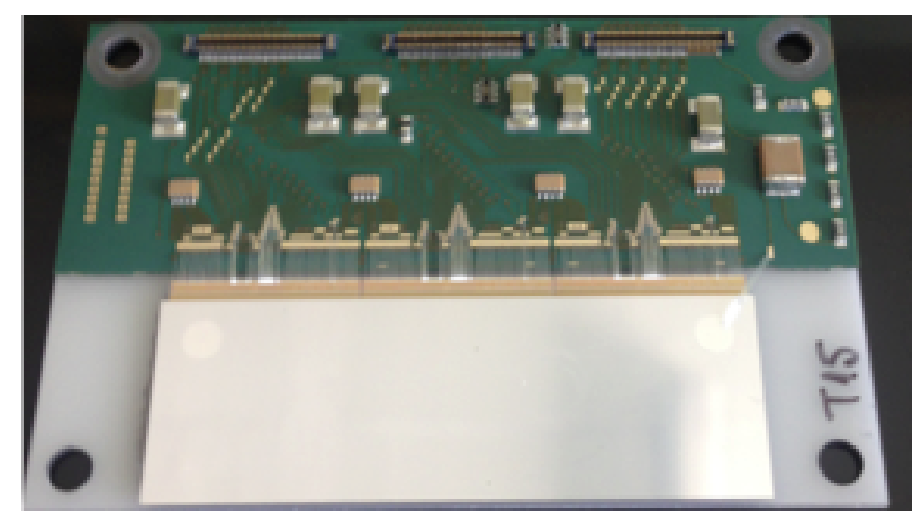

Figure 3. Photograph of a prototype triple sensor bonded to Timepix3 ASICs.

degrade the signal, reducing the signal to noise $(\mathrm{S} / \mathrm{N})$ ratio. The signal can be improved by applying higher bias voltages. Irradiated sensors are required to operate at a bias voltage of $1000 \mathrm{~V}$. This is challanging due to the non uniformity of the irradiation profile as the outer, less irradiated regions need to sustain this voltage without breakdown. For a good $\mathrm{S} / \mathrm{N}$ post irradiation, a charge collection $>6000 \mathrm{e}^{-}$is required, in addition to a uniform charge collection efficiency $>99 \%$ pre and post irradiation across the sensor. An excellent spatial resolution is also needed to maintain a good impact parameter resolution, a key parameter of the VELO performance.

To test the performance after irradiation, the sensors were sent to a range of irradiation facilities including JSI, KIT and IRRAD. They were exposed to uniform and non-uniform irradiation profiles at $4 \times 10^{15} 1 \mathrm{MeVn}_{\mathrm{eq}} / \mathrm{cm}^{2}$ (half fluence) and $8 \times 10^{15} 1 \mathrm{MeVn}_{\mathrm{eq}} / \mathrm{cm}^{2}$ the full expected lifetime fluence.

The prototype sensors are tested in both laboratory and testbeam environments. Within the laboratory the sensors are equalised and test pulse calibrations are performed. A test of the HV tolerance is carried out before the sensors are tested at testbeams.

\subsection{Timepix3 Telescope and SPS Testbeams}

For testbeam a dedicated telescope, the Timepix3 Telescope was installed at the SPS (figure 4), CERN. The telescope has 8 planes consisting of $300 \mu$ m thick p-on-n sensors bump bonded to the Timepix3 ASIC. In the centre a Device Under Test (DUT) can be installed on a motion stage allowing angular rotations and $\mathrm{x}$ and $\mathrm{y}$ translations. The DUT is mounted on a copper block that provides cooling via a peltier device and is housed in a air tight box circulating dryair. The telescope provides precise measurements of particle trajectories with a pointing resolution of $\sim 2 \mu \mathrm{m}$ and a time resolution of $\sim 1 \mathrm{~ns}$ allowing in-depth analysis of the DUT performance.

\subsection{Testbeam Analysis}

Almost 30 prototype sensors have been tested so far with further testbeams planned. A wide variety of analyses have been completed, too many to cover within the scope of this document. Therefore only a small selection will be presented. 


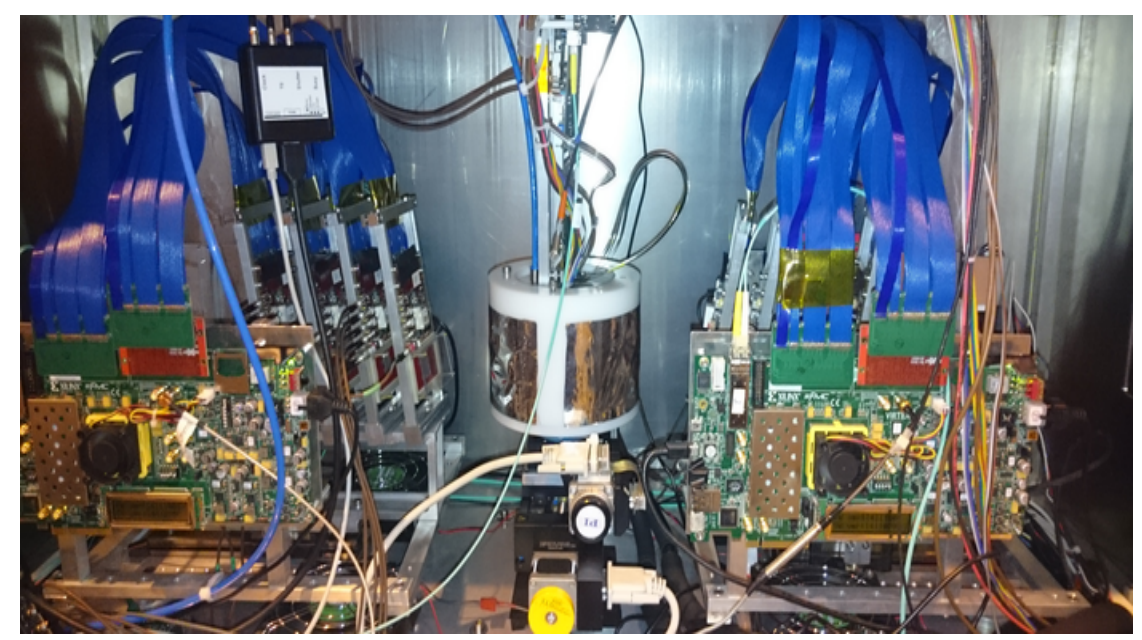

Figure 4. A photograph of the Timepix 3 Telescope installed at the SPS. There are 4 sensors on each telescope arm allowing a DUT to be installed in the centre.

\subsubsection{Charge Collection}

As discussed in section 3.1, a charge collection greater than $6000 \mathrm{e}^{-}$is required for sensors exposed to the full expected lifetime dose. Figure 5 is a plot of the collected charge as a function of bias voltage for five sensors exposed to the full fluence and one that is exposed to half the fluence. Each point gives the Most Probable Value (MPV) of a Landau fit to the charge distribution. It can be seen that both HPK and Micron sensors are able to function with a bias voltage up to $1000 \mathrm{~V}$ and recover the required charge at values $\sim 550 \mathrm{~V}$ to $\sim 750 \mathrm{~V}$ depending on the sensor. For the sensor at half fluence (pink), the required bias is less, $\sim 300 \mathrm{~V}$ as expected. This is an interesting study as it gives hints to what the required bias will be after different operational times of the experiment.

Interestingly one can investigate the charge collection as a function of the sensor depth using the grazing angle technique $[9,10]$. This is achieved by moving the DUT to large angles w.r.t. the beam, allowing the incoming particle to travel through many pixels. Knowing the number of pixels transversed, the pixel pitch and the angle of the DUT, the effective depth of the sensor can be calculated. From this the charge can be binned depending on sensor depth allowing the MPV to be determined from a Landau fit of the distribution. Figure 6 (left) shows the charge collected as a function of depth with varied applied bias. At voltages $>120 \mathrm{~V}$ the sensor is fully depleted and all the charge is collected. At lower voltages the sensor is not fully depleted, however it is seen that charge is still collected in the non-depleted region. Similarly the same plot can be made for the timing, the difference between the hit time and the time predicted by the tracking, see figure 6 (right). At voltages near full depletion the time to cross threshold is $<4 \mathrm{~ns}$. Below depletion the time for the charge to reach the threshold is much greater due the time it takes for charge to diffuse from the point where the electron-hole pairs are generated to the diffusion edge.

\subsubsection{Charge Collection Efficiency}

The charge collection efficiency is required to be $>99 \%$ and uniform across the sensor both before and after irradiation. Figure 7 (left) is a plot of the intra-pixel efficiency at $300 \mathrm{~V}$ for a irradiated 


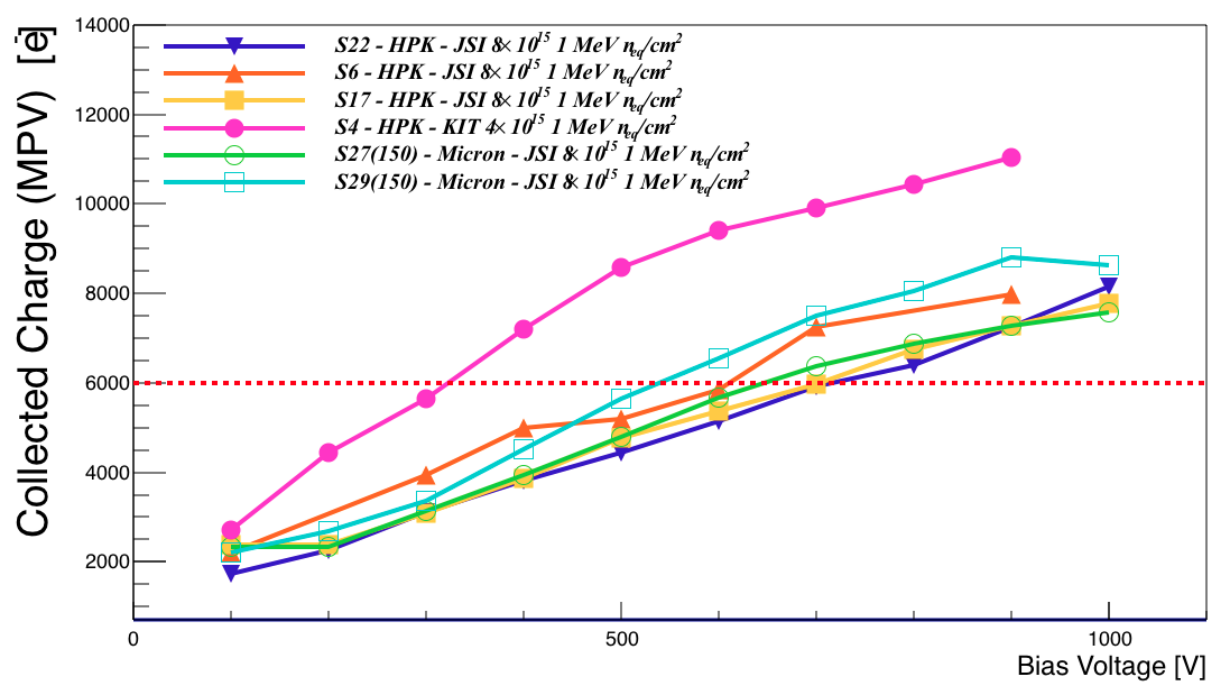

Figure 5. The collected charge as a function of bias voltage for a range of irradiated sensors from HPK and Micron. The red dashed line represents the requirement of $6000 \mathrm{e}^{-}$.
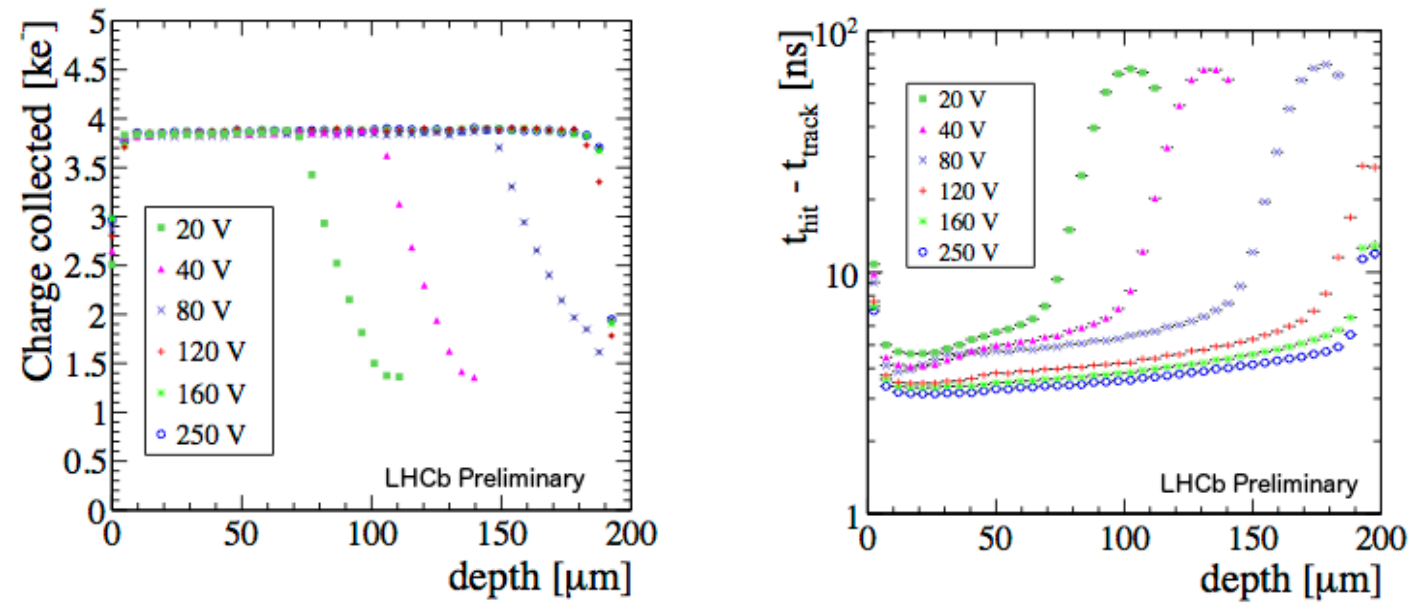

Figure 6. (left) The collected charge and the timing (right) as a function of the depth for a non-irradiated HPK sensor.

HPK sensor with an implant width of $35 \mu \mathrm{m}$. The intra-pixel efficiency is determined by splitting each pixel into sections and then superimposing the information from all pixels into one. The highest efficiency can be seen in the centre of the pixel with the efficiency decreasing towards the corners. The same trend can be seen in figure 7 (centre). However, a higher efficiency is seen over a greater area due to the larger implant width of $39 \mu \mathrm{m}$. This suggests that for the final design a larger implant would be more efficient. Figure 7 (right) is a plot of the average efficiency as a function of bias for a range of irradiated sensors. The efficiency recovers to $\sim 99 \%$ as the bias increases, with greater efficiency seen at lower bias for the sensor exposed to half fluence (green). This concludes that both HPK and Micron sensors are able to achieve the require single-hit efficiency before and after irradiation. 

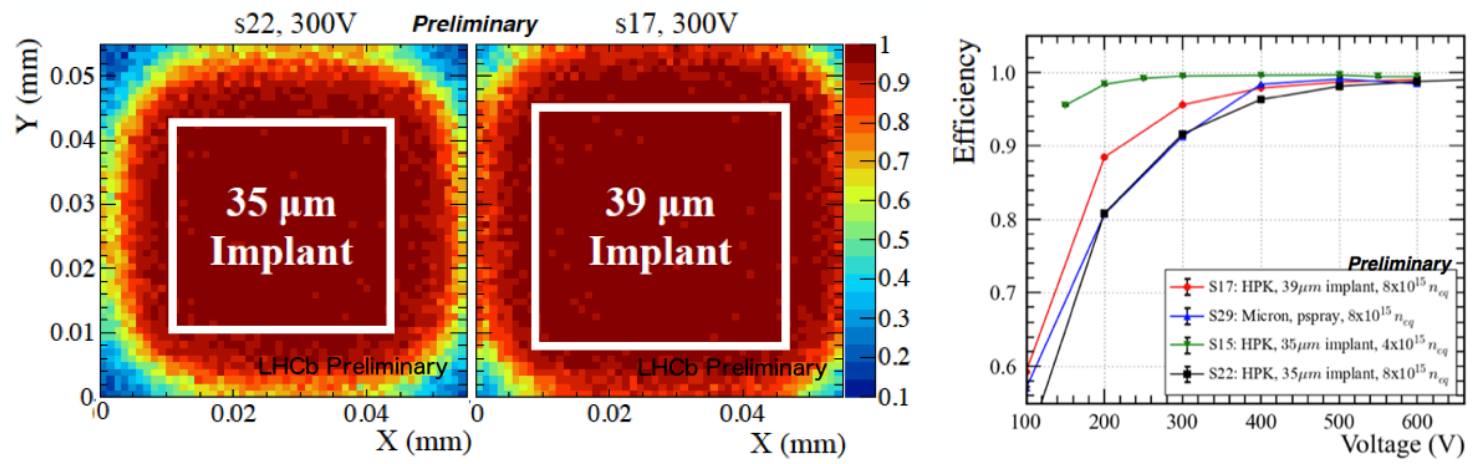

Figure 7. The intra-pixel efficiency for an irradiated HPK sensor at $300 \mathrm{~V}$ with a $35 \mu \mathrm{m}$ implant width (left) and $39 \mu \mathrm{m}$ implant width (centre). The average efficiency as a function of bias for a range of irradiated HPK and Micron sensors.

\subsubsection{Spatial Resolution}

The spatial resolution is a measure of the precision with which the position of a particle can be determined. The resolution is defined as the standard deviation of a Gaussian fit to the residual difference between the hit position and the position predicted by the track. Figure 8 shows how the resolution changes as a function of angle for a HPK and Micron sensor before and after irradiation. The resolution improves due to increased charge sharing between the pixels. At optimal angle the

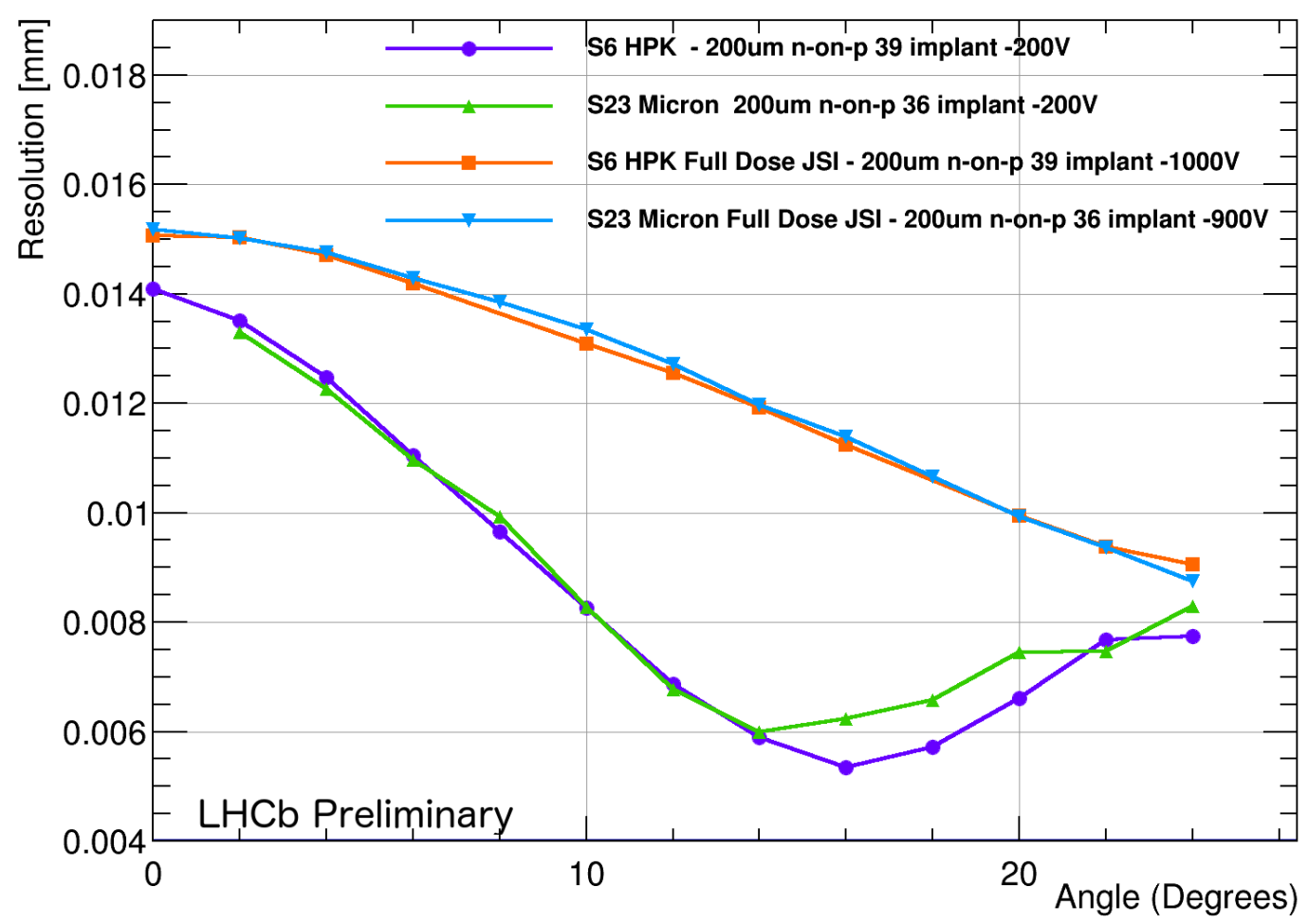

Figure 8. The spatial resolution as a function of angle for HPK and Micron sensor, before and after irradiation. 
resolution for pre-irradiated is $\sim 5 \mu \mathrm{m}$ and $\sim 9 \mu \mathrm{m}$ for irradiated, where the resolution degrades due to radiation induced charge trapping. Both the HPK and Micron sensors are seen to have an acceptable spatial resolution, both before and after irradiation.

\section{Conclusion}

The LHCb VELO will be upgraded during LS2 to allow LHCb to run at a higher luminosity. Each of the sub-components of the upgrade are progressing well. Sensor prototype testing has been ongoing since 2014 and the analysis performed has shown that most of the prototypes fulfill our requirements. Further testbeams will take place with sensors bonded to the newly arrived VeloPix. The VELO upgrade project is finishing its R\&D program and starting its construction phase.

\section{References}

[1] LHCb collaboration, The LHCb Detector at the LHC, 2008 JINST 3 S08005.

[2] The LHCb Collaboration, Letter of Intent for the LHCb Upgrade, CERN-LHCC-2011-001.

[3] T. Head, The LHCb trigger system, 2014 JINST 9 C09015.

[4] LHCb collaboration, J. Albrecht, The LHCb Trigger System: Present and Future, J. Phys. Conf. Ser. 623 (2015) 012003.

[5] R. Aaij et al., Performance of the LHCb Vertex Locator, 2014 JINST 9 C09007 [arXiv: 1405 . 7808].

[6] The LHCb Collaboration, LHCb VELO TDR: Vertex locator. Technical design report, CERN-LHCC-2001-011.

[7] The LHCb Collaboration, LHCb VELO Upgrade Technical Design Report, CERN-LHCC-2013-021.

[8] T. Poikela et al., Timepix3: a 65K channel hybrid pixel readout chip with simultaneous ToA/ToT and sparse readout, 2014 JINST 9 C05013

[9] E.J. Schioppa et al., Study of Charge Diffusion in a Silicon Detector Using an Energy Sensitivity Pixel Readout Chip, IEEE Trans. Nucl. Sci. 62 (2015) 2349.

[10] V. Chiochia et al., Simulation of heavily irradiated silicon pixel sensors and comparison with test beam measurements, IEEE Trans. Nucl. Sci. 52 (2005) 1067 [physics/0411143]. 